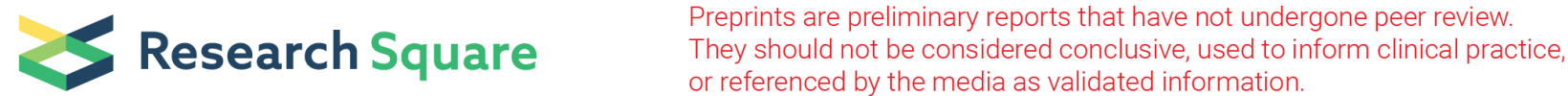

\section{Prediction of noninvasive ventilation failure in COVID-19 patients: A retrospective observational study}

Luís Neves da Silva ( $\square$ luis.neves.silva@hb.min-saude.pt )

Department of Internal Medicine, Hospital of Braga

Rui Domingues Fernandes

Department of Internal Medicine, Hospital of Braga

Ricardo Costa

Department of Internal Medicine, Hospital of Braga

Ana Andrade Oliveira

Department of Internal Medicine, Hospital of Braga

Ana Margarida Mosca

Department of Internal Medicine, Hospital of Braga

Ana Oliveira Sá

Department of Internal Medicine, Hospital of Braga

Bárbara Fontes Oliveira

Department of Internal Medicine, Hospital of Braga

Marta Braga Martins

Department of Internal Medicine, Hospital of Braga

Marta Lemos Mendes

Department of Internal Medicine, Hospital of Braga

Alexandre Carvalho

Department of Internal Medicine, Hospital of Braga

Pedro Silva Moreira

Psychological Neuroscience Laboratory, Psychology Research Center (CIPsi), School of Psychology, University of Minho

André Santa Cruz

Department of Internal Medicine, Hospital of Braga

\section{Research Article}

Keywords: COVID-19, Noninvasive ventilation, Acute respiratory failure, Ethics, Life support care, Health care rationing 
Posted Date: February 9th, 2022

DOI: https://doi.org/10.21203/rs.3.rs-1343197/v1

License: (c) (i) This work is licensed under a Creative Commons Attribution 4.0 International License. Read Full License 


\section{Abstract}

Background: In COVID-19, there are no available tools or guidelines for the difficult task of recognizing which patients do not benefit from keeping advanced respiratory support, such as noninvasive ventilation (NIV). Identifying failure is crucial to deliver the best possible care and optimize resources in overloaded healthcare systems. Therefore, this study aimed to build a model that predicts NIV failure and consequent death in COVID-19 patients.

Methods: This is a retrospective observational study with critical COVID-19 patients who needed NIV but were not candidates or did not need invasive mechanical ventilation, admitted between $1^{\text {st }}$ October 2020 and $31^{\text {st }}$ March 2021, in a tertiary Portuguese hospital. The measure of interest was NIV failure, defined as COVID-19 related in-hospital death. A binary logistic regression was performed, where the outcome (dead vs alive) was set as the dependent variable. Using the independent variables of the logistic regression a decision tree classification model was implemented.

Results: The 103 patients included had a mean age of 66.3 years old ( $S D=14.9), 38.8 \%$ were female and $82.5 \%$ autonomous. The developed prediction model was statistically significant $\left(X^{2}=119.865 ; p<.001\right)$ with an area under the curve (AUC) of 0.994. Higher age, higher number of days with increases on FiO2, higher number of days of maximum expiratory positive airway pressure, lower number of days on NIV, and lower number of days from disease onset to hospital admission were, with statistical significance, associated with increased odds of death. A decision-tree classification model was obtained to achieve the best combination of variables to predict the outcome of interest.

Conclusions: This study presents a model to predict death in COVID-19 patients treated with NIV (AUC of 0.994), including easily applicable variables that mainly reflect patients' evolution during hospitalization. Along with the decision-tree classification model, these original findings have the potential to help clinicians define the best therapeutical approach to each patient and optimize medical resources. However, further research is needed on this subject of treatment failure, not only to understand if these results are reproducible but also, in a broader sense, to help fill this gap in modern medicine guidelines.

\section{Background}

COVID-19 has affected millions of people worldwide ${ }^{1}$. Severe acute respiratory syndrome Coronavirus 2 (SARS-CoV-2) may still spread exponentially, especially when preventive measures are not fully adopted. Although most infected individuals do not require hospitalization ${ }^{2}$, we continue to observe a large group of patients who need advanced respiratory support ${ }^{3}$. The dramatic lack of ventilators felt worldwide during the COVID-19 pandemic ${ }^{4}$, confirmed, that in a setting of limited or overloaded health care systems, optimizing the use of medical resources is paramount ${ }^{5}$.

In the first months of the pandemic, the idea of early intubation was widespread, based on previous experience in acute respiratory distress syndrome (ARDS) $)^{6,7}$, which resulted in overstrained Intensive Care 
Units (ICU). Over time, other forms of respiratory support that were more available and less demanding than invasive mechanical ventilation (IMV), such as high-flow nasal oxygen (HFNO) ${ }^{8}$ and noninvasive ventilation (NIV) ${ }^{9}$, became advocated.

NIV efficacy is clearly validated in various diseases that cause respiratory failure, such as cardiogenic pulmonary edema or exacerbation of chronic obstructive pulmonary disease (COPD) ${ }^{10}$. Although controversial, use of NIV has also been supported in cases of ARDS and community acquired pneumonia, by improving clinically relevant outcomes such as progression to intubation ${ }^{11,12}$.

The use of NIV in COVID-19 was initially limited by lack of information regarding its benefit and concerns about aerosolization ${ }^{13}$. However, when it started to be used due to necessity and lack of better alternatives, studies showed an improvement in mortality rates, hospitalization time and decrease in the severity of symptoms related to COVID-19 when comparing NIV against IMV ${ }^{14}$. Moreover, no difference in mortality was found between patients who started NIV and later progressed to IMV, to those who started on $\mathrm{IMV}^{9}$. A complementary study found that patients who commenced continuous positive airway pressure (CPAP) therapy earlier had lower mortality than patients commencing CPAP later ${ }^{15}$. As more research emerged, the usage of helmet NIV instead of HFNO, was linked to a reduction in the number of patients who needed IMV ${ }^{16}$.

Due to the success of those strategies, a sequential model of care (conventional oxygen, HFNO, NIV and finally IMV) became recommended and standardized ${ }^{17}$. Optimal criteria to step-up, however, remained difficult to accomplish in most hospitals due to high influx of patients, lack of equipment or personnel. It became mandatory to allocate resources to patients that could benefit the most of them, meaning limitation of care in certain cases and recognition of treatment failure in others, especially in those not candidates for IMV. Very few studies have addressed the risk of NIV failure in COVID-19 and those who had, mainly focus on analytical data, comorbidities, age, and some clinical parameters ${ }^{18-24}$. To our knowledge, no work postulates a model to predict NIV failure in COVID-19, which includes the evolution of NIV parameters, such as the fraction of inspired oxygen (FiO2) and end positive airway pressure (EPAP), which could help guide clinical decisions.

In modern medicine, despite the high frequency of treatment failure in many scenarios, current clinical guidelines are mainly oriented to adding treatments and rarely provide support to decisions involving suspension of a therapy that has apparently become futile in a certain patient. Sadly, that is also happening in COVID-19, where studies and orientations on when to change focus and reinforce life comforting measures are scarce ${ }^{25,26}$. As sensible practitioners, we need to keep in mind that the urge to prolong a patient's life may negatively impact its quality, particularly in the last moments of it. Insisting, perhaps obsessively, on NIV when the patient will ultimately die, will lead to dysthanasia and, in a limited resource setting, preclude other patients from receiving beneficial therapies.

The main purpose of this study is to elaborate a model that, in a reliable and timely manner, predicts NIV failure in patients who are not candidates to IMV. These results may help to assess the futility of therapy, 
avoiding life prolonging measures at no benefit to the patient. After all, we were taught that first, we shall do no harm.

\section{Methods}

\section{Study design and setting}

This is a retrospective observational study with COVID-19 patients admitted between $1^{\text {st }}$ October 2020 and $31^{\text {st }}$ March 2021 in a tertiary Portuguese hospital. The hospital had an inpatient capacity of 130 beds for COVID-19 patients, of which 32 were ICU beds and 16 had continuous monitoring.

This study was approved by the Ethical Committee for Health of the Hospital of Braga (reference 61_2021).

\section{Participants' selection}

Participants were selected according to inclusion and exclusion criteria. Inclusion criteria were as follows: age $\geq 18$ years; need of NIV either as bilevel positive airway pressure (BPAP) or CPAP for more than 24 hours; initiation of the ventilatory support before the 20th day of symptoms; use of the ventilators Philips Respironics V60, Philips Respironics V60 Plus or Philips Respironics Trilogy Evo. Exclusion criteria were as follows: treatment with IMV during hospitalization; active hematological malignancy; active stage III/IV malignancy; ongoing chemotherapy; use of NIV for other respiratory conditions, without any change in the usual therapy mode or parameters; death during hospital stay not attributable to COVID-19; hospital admission due to conditions not related to COVID-19.

\section{Participants' data collection}

Patients' medical records were used to obtain baseline data on demographic features and comorbidities (age, sex, functional and cognitive status, tabagism and alcohol abuse, obesity, arterial hypertension, diabetes mellitus, dyslipidemia, heart failure, chronic liver disease, chronic kidney disease, previous stroke, immune-mediated diseases, active cancer (stages I and II), COPD, asthma, pulmonary embolism, pulmonary hypertension, other respiratory diseases, previous long term oxygen therapy), as well as information regarding the course of disease and the use of NIV during hospital stay. Details on CPAP and bilevel positive airway pressure (BPAP) settings and FiO2 were collected since the patient started NIV until the last day of its utilization. More specifically, for each 24h period of NIV, the maximum CPAP/ EPAP value (referred now on as EPAP for simplification) was collected as well as the maximum FiO2 used with that EPAP value. All data were collected and inserted into a database by the medical doctors involved in this work.

\section{Outcome measure}

The outcome measure of interest was NIV failure, defined as COVID-19 related in-hospital death. 


\section{Statistical analysis}

Descriptive statistics were obtained for sociodemographic and clinical variables. The assumption of normality was assessed with the Shapiro-Wilk test. Sociodemographic features with a prevalence higher than $10 \%$, and clinical variables, were compared between groups of patients (alive vs dead) using independent samples t-test, or the Mann-Whitney or the chi-square $\left(\mathrm{X}^{2}\right)$ test in the case of categorical variables.

Data were analysed with a binary logistic regression model, where the outcome ( 0 - alive; 1 - dead) was set as the dependent variable. The predictors of interest included age; sex; functional status; number of days from disease onset to hospital admission (when the onset of symptoms was unknown, date of diagnosis was considered) and NIV features for each patient during hospital stay: number of days on $\mathrm{NIV}$; average values of EPAP and FiO2; maximum values of EPAP and FiO2; number of days with the maximum EPAP. To reflect evolution of NIV features, two variables were created: number of days with an increase of EPAP and number of days with an increase of FiO2. The area under the curve (AUC), sensitivity and specificity were extracted as performance metrics of this model.

A decision-tree classification model was implemented to further explore the combination of variables that best predicts the outcome of interest. To achieve this, the same variables defined as independent variables in the logistic regression model were entered as features of interest. With the decision-tree classification model, we aim to divide the dataset of alive and non-alive patients into homogeneous groups, based on successive decision boundaries established according to relevant features that minimize the probability of a random element to be incorrectly classified (the Gini impurity).

Descriptive statistics and logistic regression analysis were performed using computer software JASP (Version 0.16$)^{27}$. The decision-tree classification model was implemented with Scikit-learn (Version 1.0.1 $)^{28}$. Statistical significance was defined at the $p<.05$ level.

\section{Results}

\section{Patients Characteristics at Hospital Admission and Group Differences}

From 1st October 2020 to 31st March 2021, 139 patients treated with NIV were identified, of which 103 met inclusion and exclusion criteria and were eligible to analysis. Thirty-nine patients (37.9\%) died during hospital stay due to COVID-19 (Figure 1).

Mean age was 66.3 years old $(S D=14.9)$, and 40 patients $(38.8 \%)$ were women. No patient was vaccinated against COVID-19. Patients who died were significantly older $\left(M_{\text {alive }}=60.4, S_{\text {alive }}=14.0\right.$; $\left.\mathrm{M}_{\text {dead }}=76.1, \mathrm{SD}_{\text {dead }}=10.8 ; \mathrm{p}<.001\right)$. Arterial hypertension was the most common comorbidity $(71.8 \%)$ followed by dyslipidemia (52.4\%), obesity (35.9\%), diabetes mellitus (31.1\%) and heart failure (16.5\%). Eighty-five patients (82.5\%) were autonomous for daily activities. Patients with arterial hypertension $\left(X^{2}=5.061 ; p=.02\right)$, dyslipidemia $\left(X^{2}=7.106 ; p=.008\right)$, heart failure $\left(X^{2}=12.706 ; p<.001\right)$, a 
compromised cognitive status $\left(X^{2}=6.678 ; p=.01\right)$ and that were non autonomous $\left(X^{2}=14.769 ; p<.001\right)$, were more likely to die.

Patients presented to hospital from disease onset in a median time of 6.0 days (IQR=3.5-8.0). The median time to presentation was significantly lower in patients who died comparing to those who remained alive $\left(\mathrm{Mdn}_{\text {alive }}=7.0, \mathrm{IQR}_{\text {alive }}=5.0-9.0 ; \mathrm{Mdn}_{\text {dead }}=4.0, \mathrm{IQR}_{\text {dead }}=0.0-6.0 ; \mathrm{p}<.001\right)$ as well as the length of stay in the hospital $\left(\mathrm{Mdn}_{\text {alive }}=17.0, \mathrm{IQR}_{\text {alive }}=13.0-26.0 ; \mathrm{Mdn}_{\text {dead }}=9.0, \mathrm{IQR}_{\text {dead }}=6.0-12.0 ; \mathrm{p}<\right.$ $.001)$.

More detailed information on the population's characteristics at hospital admission is shown on Table 1. 
Table 1. Patients Characteristics and Group Differences

\begin{tabular}{|lllll} 
Characteristic & $\begin{array}{l}\text { Total } \\
(n=103)\end{array}$ & $\begin{array}{l}\text { Dead } \\
(n=39)\end{array}$ & $\begin{array}{l}\text { Alive } \\
(n=64)\end{array}$ & $\begin{array}{l}\text { Group } \\
\text { comparison }\end{array}$ \\
\hline Age, mean (SD), y & $66.3(14.9)$ & $76.1(10.8)$ & $60.4(14.0)$ & $\begin{array}{l}t(101)=-6.011 ; \\
p<.001\end{array}$
\end{tabular}

Sex, No. (\%)

Female

Male

Functional Status, No. (\%)

Autonomous

Non-autonomous

Cognitive Status, No. (\%)

\begin{tabular}{|c|c|c|c|c|}
\hline Preserved & $94(91.3 \%)$ & $32(82.1 \%)$ & $62(96.9 \%)$ & \multirow{2}{*}{$\begin{array}{l}X^{2}=6.678 ; p= \\
.01\end{array}$} \\
\hline Compromised & $9(8.7 \%)$ & $7(17.9 \%)$ & $2(3.1 \%)$ & \\
\hline Obesity, No. (\%) & $37(35.9 \%)$ & $11(28.2 \%)$ & $26(40.6 \%)$ & $\begin{array}{l}X^{2}=1.624 ; p= \\
.20\end{array}$ \\
\hline Arterial Hypertension, No. (\%) & $74(71.8 \%)$ & $33(84.6 \%)$ & $41(64.1 \%)$ & $\begin{array}{l}X^{2}=5.061 ; p= \\
.02\end{array}$ \\
\hline Diabetes Mellitus, No. (\%) & $32(31.1 \%)$ & $14(35.9 \%)$ & $18(28.1 \%)$ & $\begin{array}{l}X^{2}=0.684 ; p= \\
.41\end{array}$ \\
\hline Dyslipidemia, No. (\%) & $54(52.4 \%)$ & $27(69.2 \%)$ & $27(42.2 \%)$ & $\begin{array}{l}X^{2}=7.106 ; p= \\
.008\end{array}$ \\
\hline \multicolumn{5}{|l|}{ Tabagism, No. (\%) } \\
\hline Previous & $7(6.8 \%)$ & $3(7.7 \%)$ & $4(6.3 \%)$ & \\
\hline Current & $3(2.9 \%)$ & $1(2.6 \%)$ & $2(3.1 \%)$ & \\
\hline \multicolumn{5}{|l|}{ Alcohol abuse, No. (\%) } \\
\hline Previous & $6(5.8 \%)$ & $3(7.7 \%)$ & $3(4.7 \%)$ & \\
\hline Current & $5(4.9 \%)$ & $2(5.1 \%)$ & $3(4.7 \%)$ & \\
\hline \multicolumn{5}{|c|}{ Immune-mediated diseases, №. (\%) } \\
\hline Still's disease & $1(1.0 \%)$ & $0(0.0 \%)$ & $1(1.6 \%)$ & \\
\hline Rheumatoid Arthritis & $1(1.0 \%)$ & $0(0.0 \%)$ & $1(1.6 \%)$ & \\
\hline
\end{tabular}

$85(82.5 \%) \quad 25(64.1 \%) \quad 60(93.7 \%) \quad X^{2}=14.769 ; p<$

$18(17.5 \%) \quad 14(35.9 \%) \quad 4(6.3 \%)$ .001 
Table 1. Patients Characteristics and Group Differences

\begin{tabular}{|c|c|c|c|c|}
\hline Characteristic & $\begin{array}{l}\text { Total } \\
(n=103)\end{array}$ & $\begin{array}{l}\text { Dead } \\
(n=39)\end{array}$ & $\begin{array}{l}\text { Alive } \\
(\mathrm{n}=64)\end{array}$ & $\begin{array}{l}\text { Group } \\
\text { comparison }\end{array}$ \\
\hline Psoriasis & $1(1.0 \%)$ & $1(2.6 \%)$ & $0(0.0 \%)$ & \\
\hline Inflammatory Bowel Disease & $1(1.0 \%)$ & $0(0.0 \%)$ & $1(1.6 \%)$ & \\
\hline Immune Thrombocytopenia & $1(1.0 \%)$ & $1(2.6 \%)$ & $0(0.0 \%)$ & \\
\hline Stage I/II Active Cancer, No. (\%) & $0(0.0 \%)$ & $0(0.0 \%)$ & $0(0.0 \%)$ & \\
\hline COPD, No. (\%) & $7(6.8 \%)$ & $3(7.7 \%)$ & $4(6.3 \%)$ & \\
\hline Asthma, №. (\%) & $3(2.9 \%)$ & $1(2.6 \%)$ & $2(3.1 \%)$ & \\
\hline Obstructive Sleep Apnea, No. (\%) & $9(8.7 \%)$ & $4(10.3 \%)$ & $5(7.8 \%)$ & \\
\hline Hypersensitivity Pneumonitis, No. (\%) & $1(1.0 \%)$ & $1(2.6 \%)$ & $0(0.0 \%)$ & \\
\hline PH, No. (\%) & $1(1.0 \%)$ & $1(2.6 \%)$ & $0(0.0 \%)$ & \\
\hline Previous PE, No. (\%) & $0(0.0 \%)$ & $0(0.0 \%)$ & $0(0.0 \%)$ & \\
\hline LTOT, No. (\%) & $1(1.0 \%)$ & $0(0.0 \%)$ & $1(1.6 \%)$ & \\
\hline HF, No. (\%) & $17(16.5 \%)$ & $14(35.9 \%)$ & $5(7.8 \%)$ & $\begin{array}{l}X^{2}=12.706 ; p< \\
.001\end{array}$ \\
\hline Previous Stroke, No. (\%) & $6(5.6 \%)$ & $6(15.4 \%)$ & $0(0.0 \%)$ & \\
\hline \multicolumn{5}{|l|}{ CLD, No. (\%) } \\
\hline Child A & $2(1.9 \%)$ & $1(2.6 \%)$ & 1 (1.6\%) & \\
\hline Child B & $0(0.0 \%)$ & $0(0.0 \%)$ & $0(0.0 \%)$ & \\
\hline Child C & $0(0.0 \%)$ & $0(0.0 \%)$ & $0(0.0 \%)$ & \\
\hline \multicolumn{5}{|l|}{ CKD, No. (\%) } \\
\hline G3 & $7(6.8 \%)$ & $5(12.8 \%)$ & $2(3.1 \%)$ & \\
\hline G4 & $0(0.0 \%)$ & $0(0.0 \%)$ & $0(0.0 \%)$ & \\
\hline G5 & $2(1.9 \%)$ & $2(5.1 \%)$ & $0(0.0 \%)$ & \\
\hline $\begin{array}{l}\mathrm{PaO} 2 / \mathrm{FiO} 2 \text { ratio at admission, median } \\
\text { (IQR) }\end{array}$ & $\begin{array}{l}204.0 \\
(114.0- \\
247.3)\end{array}$ & $\begin{array}{l}212.0 \\
(131.5- \\
254.0)\end{array}$ & $\begin{array}{l}195.5 \\
(108.4- \\
247.2)\end{array}$ & $\begin{array}{l}U(101)=1180 ; \\
p=.65\end{array}$ \\
\hline $\begin{array}{l}\text { Days from disease onset to hospital } \\
\text { admission, median (IQR) }\end{array}$ & $\begin{array}{l}6.0(3.5- \\
8.0)\end{array}$ & $\begin{array}{l}4.0(0.0- \\
6.0)\end{array}$ & $\begin{array}{l}7.0(5.0- \\
9.0)\end{array}$ & $\begin{array}{l}U(101)=1852 ; \\
p<.001\end{array}$ \\
\hline
\end{tabular}


Table 1. Patients Characteristics and Group Differences

\begin{tabular}{lllll}
\hline Characteristic & $\begin{array}{l}\text { Total } \\
(\mathbf{n}=103)\end{array}$ & $\begin{array}{l}\text { Dead } \\
(\mathbf{n}=39)\end{array}$ & $\begin{array}{l}\text { Alive } \\
(\mathbf{n}=64)\end{array}$ & $\begin{array}{l}\text { Group } \\
\text { comparison }\end{array}$ \\
\hline Length of stay, median (IQR) & $13.0(9.0-$ & $9.0(6.0-$ & $17.0(13.0-$ & $\mathrm{U}(101)=$ \\
& $21.3)$ & $12.0)$ & $26.0)$ & $1942.5 ; \mathrm{p}<$ \\
& & & & .001
\end{tabular}

Abbreviations: COPD, chronic obstructive pulmonary disease; CKD, chronic kidney disease; CLD, chronic liver disease; HF, heart failure; LTOT, long-term oxygen therapy; PE, pulmonary embolism; PH, pulmonary hypertension

${ }^{\mathrm{a}}$ No. $=$ numbers of patients included in the analysis.

\section{NIV features and evolution during hospital stay}

The median time on NIV was 6.0 days (IQR= 4.0-9.0 days) without a significant difference between patients who died or survived $\left(\mathrm{Mdn}_{\text {alive }}=6.0, \mathrm{IQR}_{\text {alive }}=4.0-9.3 ; \mathrm{Mdn}_{\text {dead }}=7.0, \mathrm{IQR}_{\text {dead }}=4.5-9.0 ; \mathrm{p}=.62\right)$.

Patients who died had significantly higher average values of EPAP $\left(\mathrm{Mdn}_{\text {alive }}=10.0, \mathrm{IQR}_{\text {alive }}=9.1-10.5\right.$; $\left.\mathrm{Mdn}_{\text {dead }}=11.0, \mathrm{IQR}_{\text {dead }}=10.3-12.0 ; \mathrm{p}<.001\right)$ and of maximum EPAP $\left(\mathrm{Mdn}_{\text {alive }}=12.0, \operatorname{IQR}_{\text {alive }}=10.0-12.0\right.$; $\left.M d n_{\text {dead }}=12.0, I Q R_{\text {dead }}=12.0-12.0 ; p<.001\right)$ as well as a higher number of days on maximum EPAP $\left(\mathrm{Mdn}_{\text {alive }}=1.0, \mathrm{IQR}_{\text {alive }}=1 \cdot 0-1 \cdot 0 ; \mathrm{Mdn}_{\text {dead }}=2 \cdot 0, \mathrm{IQR}_{\text {dead }}=1 \cdot 0-2 \cdot 0 ; \mathrm{p}=.002\right)$. Patients who died had significantly higher average values of FiO2 $\left(\mathrm{Mdn}_{\text {alive }}=65.0, \mathrm{IQR}_{\mathrm{alive}}=60.6-68.6 ; \mathrm{Mdn}_{\text {dead }}=85.0\right.$, IQR $\mathrm{R}_{\text {dead }}=$ 77.5-91.5; $p<.001)$ and of maximum FiO2 ( $\mathrm{Mdn}_{\text {alive }}=75.0, \mathrm{IQR}_{\text {alive }}=68.8-81.3 ; \mathrm{Mdn}_{\text {dead }}=100.0, \mathrm{IQR}_{\text {dead }}=$ 90.0-100.0; $p<.001)$. The number of days with increases on EPAP was also significantly higher on those who died ( $\left.\mathrm{Mdn}_{\text {alive }}=0.0, \mathrm{IQR}_{\text {alive }}=0.0-1.0 ; \mathrm{Mdn}_{\text {dead }}=1.0, \mathrm{IQR}_{\text {dead }}=0.0-2.0 ; \mathrm{p}=.007\right)$ as it was the number of days with increases on FiO2 ( $\left.\mathrm{Mdn}_{\text {alive }}=1.0, \mathrm{IQR}_{\mathrm{alive}}=0.0-2.0 ; \mathrm{Mdn}_{\text {dead }}=1.0, \mathrm{IQR}_{\text {dead }}=1.0-3.0 ; \mathrm{p}=.005\right)$. These and other results are provided in Table 2. 
Table 2. Group differences regarding NIV features related variables

\begin{tabular}{|c|c|c|c|c|}
\hline Characteristics & $\begin{array}{l}\text { Total } \\
(n=103)\end{array}$ & $\begin{array}{l}\text { Dead } \\
(n=39)\end{array}$ & $\begin{array}{l}\text { Alive } \\
(n=64)\end{array}$ & $\begin{array}{l}\text { Group } \\
\text { comparison }\end{array}$ \\
\hline Days on NIV, median (IQR) (min-max) & $\begin{array}{l}6.0(4.0- \\
9.0)(2-28)\end{array}$ & $\begin{array}{l}7.0(4.5- \\
9.0)(2-23)\end{array}$ & $\begin{array}{l}6.0(4.0- \\
9.3)(2-28)\end{array}$ & $\begin{array}{l}U(101)= \\
1174.5 ; p=.62\end{array}$ \\
\hline Average EPAP, median (IQR) & $\begin{array}{l}10.2(9.5- \\
11.2)\end{array}$ & $\begin{array}{l}11.0(10.3- \\
12.0)\end{array}$ & $\begin{array}{l}10.0(9.1- \\
10.5)\end{array}$ & $\begin{array}{l}U(101)=633.0 \\
p<.001\end{array}$ \\
\hline Maximum EPAP, median (IQR) & $\begin{array}{l}12.0(10.0- \\
12.0)\end{array}$ & $\begin{array}{l}12.0(12.0- \\
12.0)\end{array}$ & $\begin{array}{l}12.0(10.0- \\
12.0)\end{array}$ & $\begin{array}{l}U(101)=781.0 \\
p<.001\end{array}$ \\
\hline $\begin{array}{l}\text { Days with maximum EPAP, median } \\
\text { (IQR) }\end{array}$ & $\begin{array}{l}1.0(1.0- \\
2.0)\end{array}$ & $\begin{array}{l}2.0(1.0- \\
2.0)\end{array}$ & $\begin{array}{l}1.0(1.0- \\
1.0)\end{array}$ & $\begin{array}{l}U(101)=857.5 \\
p=0.002\end{array}$ \\
\hline Average FiO2, median (IQR) & $\begin{array}{l}68.3(62.6- \\
81.2)\end{array}$ & $\begin{array}{l}85.0(77.5- \\
91.5)\end{array}$ & $\begin{array}{l}65.0(60.6- \\
68.6)\end{array}$ & $\begin{array}{l}U(101)=344.5 \\
p<.001\end{array}$ \\
\hline Maximum FiO2, median (IQR) & $\begin{array}{l}80.0(70.0- \\
100.0)\end{array}$ & $\begin{array}{l}100.0 \\
(90.0- \\
100.0)\end{array}$ & $\begin{array}{l}75.0(68.8- \\
81.3)\end{array}$ & $\begin{array}{l}U(101)=489.5 \\
p<.001\end{array}$ \\
\hline $\begin{array}{l}\text { Days with conventional oxygen } \\
\text { before NIV, median (IQR) }\end{array}$ & $\begin{array}{l}1.0(0.0- \\
2.0)\end{array}$ & $\begin{array}{l}1.0(0.0- \\
2.0)\end{array}$ & $\begin{array}{l}0.0(0.0- \\
2.0)\end{array}$ & $\begin{array}{l}U(101)= \\
1114.0 ; p=0.33\end{array}$ \\
\hline $\begin{array}{l}\text { Days with HFNO before NIV, median } \\
\text { (IQR) }\end{array}$ & $\begin{array}{l}0.0(0.0- \\
1.0)\end{array}$ & $\begin{array}{l}0.0(0.0- \\
1.0)\end{array}$ & $\begin{array}{l}0.0(0.0- \\
1.0)\end{array}$ & $\begin{array}{l}U(101)= \\
1275.0 ; p=0.84\end{array}$ \\
\hline $\begin{array}{l}\text { Days with EPAP increases, median } \\
\text { (IQR) }\end{array}$ & $\begin{array}{l}1.0(0.0- \\
1.5)\end{array}$ & $\begin{array}{l}1.0(0.0- \\
2.0)\end{array}$ & $\begin{array}{l}0.0(0.0- \\
1.0)\end{array}$ & $\begin{array}{l}U(101)=879.5 \\
p=0.007\end{array}$ \\
\hline $\begin{array}{l}\text { Days with FiO2 increases, median } \\
\text { (IQR) }\end{array}$ & $\begin{array}{l}1.0(0.0- \\
3.0)\end{array}$ & $\begin{array}{l}1.0(1.0- \\
3.0)\end{array}$ & $\begin{array}{l}1.0(0.0- \\
2.0)\end{array}$ & $\begin{array}{l}\mathrm{U}(101)=857.5 \\
\mathrm{p}=0.005\end{array}$ \\
\hline
\end{tabular}

Abbreviations: EPAP, end positive airway pressure; FiO2, fraction of inspired oxygen; HFNO, high-flow nasal oxygen; NIV, noninvasive ventilation.

\section{Logistic regression model}

Due to the high correlation between average FiO2 and maximum FiO2 values $(r(101)=0.713 ; p<.001)$ as well as average EPAP and maximum EPAP $(r(101)=0.807 ; p<.001)$, only the average values of EPAP and FiO2 were retained in the model. As the main purpose was to build a model based on NIV features, comorbidities that were prevalent and associated with death in our sample (arterial hypertension, dyslipidemia, and heart failure) were not included, also serving to lessen the risk of overfitting the model.

The logistic regression model was statistically significant $\left(X^{2}=119.865 ; p<.001\right)$ with an AUC of 0.994 , a sensitivity of 0.974 and a specificity of 0.969 . The model's receiver operating characteristic (ROC) curve is shown on Figure 2. 
Increased age $(\mathrm{OR}=1.334 ; 95 \% \mathrm{Cl}[0.073,0.503] ; \mathrm{p}=.009)$, a higher number of days with increases on FiO2 (OR= 120.176; $95 \% \mathrm{Cl}[1.447,8.131] ; \mathrm{p}=.005)$, a lower number of days on NIV (OR= $0.180 ; 95 \% \mathrm{Cl}$ $[-2.944,-0.482] ; p=.006)$, a higher number of days with EPAP at maximum values (OR $=33.469 ; 95 \% \mathrm{Cl}$ $[0.442,6.580] ; p=.03)$ and a lower number of days from disease onset to hospital admission ( $O R=0.432$; $95 \% \mathrm{Cl}[-1.549,-0.129] ; \mathrm{p}=0.02)$ were associated with increased odds of death (Table 3).

Table 3. Binary Logistic Regression

Wald Test

$95 \%$

Confidence

interval

\begin{tabular}{lccccccccc}
\hline & Estimate & Standard & Odds & z & Wald & df & p & Lower & Upper \\
& & Error & Ratio & & Statistic & & bound & bound \\
\hline (Intercept) & -40.827 & 15.975 & $1.858 \mathrm{e}-$ & -2.556 & 6.531 & 1 & 0.01 & -72.138 & -9.516 \\
& & & 18 & & & & & & \\
\hline Sex & 2.090 & 1.653 & 8.083 & 1.264 & 1.598 & 1 & 0.21 & -1.150 & 5.329 \\
\hline Age & 0.288 & 0.110 & 1.334 & 2.629 & 6.910 & 1 & 0.009 & 0.073 & 0.503 \\
\hline Functional Status & 3.162 & 1.967 & 23.617 & 1.607 & 2.584 & 1 & 0.11 & -0.693 & 7.017 \\
\hline Days with FiO2 increases & 4.789 & 1.705 & 120.176 & 2.809 & 7.890 & 1 & 0.005 & 1.447 & 8.131 \\
\hline Average EPAP & 1.307 & 0.884 & 3.696 & 1.478 & 2.186 & 1 & 0.14 & -0.426 & 3.040 \\
\hline Average FiO2 & 0.131 & 0.071 & 1.140 & 1.836 & 3.370 & 1 & 0.07 & -0.009 & 0.270 \\
\hline Days on NIV & -1.713 & 0.628 & 0.180 & -2.727 & 7.435 & 1 & 0.006 & -2.944 & -0.482 \\
\hline Days with maximum EPAP & 3.511 & 1.566 & 33.469 & 2.242 & 5.027 & 1 & 0.03 & 0.442 & 6.580 \\
\hline Days from disease onset to hospital & -0.839 & 0.362 & 0.432 & -2.316 & 5.364 & 1 & 0.02 & -1.549 & -0.129 \\
admission & & & & & & & & \\
\hline
\end{tabular}

Abbreviations: EPAP, end positive airway pressure; FiO2, fraction of inspired oxygen; NIV, noninvasive ventilation.

Abbreviations: EPAP, end positive airway pressure; FiO2, fraction of inspired oxygen; NIV, noninvasive ventilation.

\section{Decision tree classification model}

The model represented in Figure 3 starts with a root node in which all the observations $\left(n_{\text {total }}=103 ; n_{\text {alive }}=\right.$ $64 ; n_{\text {dead }}=39$ ) are present. The first decision boundary was based on the average FiO2 values: approximately $82 \%$ of the patients who die have average $\mathrm{FiO} 2$ values greater than $72.6 \%$. A new decision 
boundary is defined by age in the group of patients with average FiO2 values equal or lower than $72.6 \%$. From a total of 7 patients in this branch, 6 patients with age greater than 74 years old die. Among the group of patients with average $\mathrm{FiO} 2$ values greater than $72.6 \%$, from a total of 32 patients, 30 patients with EPAP average values greater than 9.9 die. Third level decision boundaries are established according to $\mathrm{FiO} 2$ average values, number of days with increases on FiO2, number of days between disease onset and hospitalization and the total number of days on NIV.

\section{Discussion}

In the sample of this work, a mortality rate of $37.9 \%$ was observed. Patients had a mean age of 66.3 years old and $61.2 \%$ were males. Arterial hypertension was the most common comorbidity $(71.8 \%)$ and there was a high prevalence of other cardiometabolic comorbidities. Overall, this is in accordance with other studies with COVID-19 patients treated with NIV29.

It should come as no surprise that patients who died were significantly older ${ }^{30}$ and had a worse functional status ${ }^{31}$. These two factors alone can be easily determined on admission and provide a basis for predicting patient response to NIV. Patients who died had a lower number of days on NIV which may seem surprising at first sight. Revising our data, $71 \%$ of those who died were treated for a period of less than 5 days and $50 \%$ of patients of them were older than 70 years old. Moreover, patients who died had significant differences in several variables that reflect the need for greater ventilatory support: higher average $\mathrm{FiO} 2$ and EPAP, higher maximum $\mathrm{FiO} 2$ and EPAP as well as higher number of days with increases on FiO2 and EPAP and higher number of days with EPAP on maximum values. This may reflect a rapidly progressive disease course in a large group of patients. Survivors had perhaps a slower progression of disease, were able to control inflammation and reach a plateau before their condition started to improve. In COVID-19, the recovery process is usually slow, perhaps justifying why survivors had more days under NIV. This explanation also elucidates why patients who died had significantly less time from disease onset to hospital admission, that is, it may reflect a more aggressive inflammatory response, marking a worse clinical trajectory, as it has been suggested ${ }^{32}$.

A logistic regression model, that was statistically significant with an AUC of 0.994 a sensitivity of 0.974 and a specificity of 0.969 , was obtained. Based on the variables used on the logistic regression model, a decision tree was developed, which allows the medical team to quickly stratify a patient into a group of similar patients and extrapolate mortality rates. For instance, it is striking that a mortality of $93.8 \%$ is expected in a group of patients with average $\mathrm{FiO} 2$ greater than $72.6 \%$ and average EPAP greater than 9.9 . We can further stratify the risk by continuing down the decision tree, but such a high mortality rate will necessarily promote a clinical discussion about the patient's best interest and the most adequate therapeutic approach.

Clinicians have progressively more data and tools available to predict a patient's prognosis. For example, Ling Liu, et $\mathrm{al}^{24}$ have found several parameters in their study that also help predict NIV failure and were able to develop an online calculator to assist decision making. Works like this have been mainly limited to 
predict NIV failure based on analytical parameters and comorbidities. While this kind of predictors are undoubtedly important, our model was intentionally built upon variables that reflect the evolution throughout hospital stay, making this work innovative, useful to clinical practice and helpful on filling a gap in literature. Overall, works of this kind may become crucial in settings of scarce medical resources, be it from chronic lack of resources or from unexpected demand. Accurate assessment of a patient prognosis allows to better employ resources, treating those who may probably benefit from continuation of advanced therapeutic measures, and to give more thought and time to life comforting measures which are often regrettably omitted. It must be stated, however, that the results provided should not override clinical sense or wisdom.

\section{Limitations}

This study has a retrospective observational design, which does not allow to rule out residual confounding, and has an inferior level of evidence. Data was collected from medical records, which are prone to human error and are not always complete or updated.

Most patients initiated NIV when HFNO failed to maintain a $\mathrm{PaO} 2>60 \mathrm{mmHg}$ with a FiO2> $70 \%$. Progression to IMV was based on clinical judgement, case by case, and was not always consistent throughout the pandemic, which may have interfered with sample selection. All patients received the standard of care with dexamethasone, with none receiving other agents such as remdesivir, tocilizumab or antibiotics. These practices can differ from other centers, making it hard to extrapolate our findings. Similarly, centres with access to distinct models of ventilators which perform differently may experience non-identical results. We may also hypothesize that a sample of fully vaccinated individuals could have shown different mortality rates and patterns.

Finally, the number of patients in our center made it impossible to have a separate cohort for crossvalidation of the model, a technique to lessen the risk of overfitting, which ideally is done with samples from other centers.

\section{Conclusion}

This retrospective observational study, despite its limitations, presents a model to predict NIV failure, defined as COVID-19 related in-hospital death, in patients who are not candidate to IMV, with an AUC of 0.994 and based on easily applicable variables that also reflect evolution during hospital stay (i.e. Sex; Age; Functional Status; Days from disease onset to hospital admission; Days on NIV; Average FiO2; Days with FiO2 increases; Average EPAP; Days with maximum EPAP), that was further explored with the implementation of a decision-tree classification model, allowing for the best combination of variables to predict mortality. These findings help clinical decision making as to optimize medical resources and prioritize life comforting measures when adequate, hopefully preventing dysthanasia.

\section{Declarations}


Ethics approval and consent to participate: This study was approved by the Ethical Committee for Health of the Hospital of Braga (reference 61_2021).

Consent for publication: Not applicable.

Availability of data and materials: The datasets used and/or analysed during the current study are available from the corresponding author on reasonable request.

Competing interests: The authors declare that they have no competing interests.

Funding: This research did not receive any specific grant from funding agencies in the public, commercial, or not-for-profit sectors.

\section{Authors' contributions:}

LNS and RDF contributed equally to this manuscript and should be considered co-first authors.

LNS, conception of the work, acquisition of data, analysis and interpretation of data for the work, drafting the work, and revision for important intellectual content. RDF, conception of the work, acquisition of data and interpretation of data for the work, drafting the work, and revision for important intellectual content. RC, acquisition of data and interpretation of data for the work, drafting the work, and revision for important intellectual content. AAO, acquisition of data and revision for important intellectual content. AMM, acquisition of data and revision for important intellectual content. AOS, acquisition of data and revision for important intellectual content. BO, acquisition of data and revision for important intellectual content. MBM, acquisition of data and revision for important intellectual content. MLM, acquisition of data and revision for important intellectual content. AC, conception of the work and revision for important intellectual content. PSM, conception of the work, analysis and interpretation of data for the work and revision for important intellectual content. ASC, supervision, conception of the work and revision for important intellectual content. All authors reviewed and approved the final manuscript.

Acknowledgements: Not applicable.

\section{Abbreviations}

ARDS - Acute Respiratory Distress Syndrome

AUC - Area Under the Curve

BPAP - Bilevel Positive Airway Pressure

COPD - Chronic Obstructive Pulmonary Disease

CPAP - Continuous Positive Airway Pressure

EPAP - End Positive Airway Pressure 
FiO2 - Fraction of Inspired Oxygen

HFNO - High-Flow Nasal Oxygen

ICU - Intensive Care Units

IMV - Invasive Mechanical ventilation

NIV - Noninvasive Ventilation

ROC - Receiver Operating Characteristic

SARS-CoV-2 - Severe Acute Respiratory Syndrome Coronavirus 2

\section{References}

1. Ritchie H, Mathieu E, Rodés-Guirao L, Appel C, Giattino C, Ortiz-Ospina E, et al. Coronavirus Pandemic (COVID-19). Our World in Data. (2000) https://ourworldindata.org/coronavirus. Accessed October 11, 2021.

2. COVID-19 hospitalization and emergency department statistics. Cihi.ca.

https://www.cihi.ca/en/covid-19-hospitalization-and-emergency-department-statistics. Accessed October $11,2021$.

3. Yang X, Yu Y, Xu J, Shu H, Xia J, Liu H, et al. Clinical course and outcomes of critically ill patients with SARS-CoV-2 pneumonia in Wuhan, China: a single-centered, retrospective, observational study. Lancet Respir Med. 2020;8(5):475-81.

4. Dar M, Swamy L, Gavin D, Theodore A. Mechanical-ventilation supply and options for the COVID19 pandemic. Leveraging all available resources for a limited resource in a crisis. Ann Am Thorac Soc. $2021 ; 18(3): 408-16$.

5. Emanuel EJ, Persad G, Upshur R, Thome B, Parker M, Glickman A, et al. Fair allocation of scarce medical resources in the time of covid-19. N Engl J Med. 2020;382(21):2049-55.

6. Nava S, Hill N. Non-invasive ventilation in acute respiratory failure. Lancet. 2009;374(9685):250-9.

7. Rochwerg B, Brochard L, Elliott MW, Hess D, Hill NS, Nava S, et al. Official ERS/ATS clinical practice guidelines: noninvasive ventilation for acute respiratory failure. Eur Respir J. 2017;50(2).

8. Teng X-B, Shen Y, Han M-F, Yang G, Zha L, Shi J-F. The value of high-flow nasal cannula oxygen therapy in treating novel coronavirus pneumonia. Eur J Clin Invest. 2021;51(3):e13435.

9. Daniel P, Mecklenburg M, Massiah C, Joseph MA, Wilson C, Parmar P, et al. Non-invasive positive pressure ventilation versus endotracheal intubation in treatment of COVID-19 patients requiring 
ventilatory support. Am J Emerg Med. 2021;43:103-8.

10. Mateos-Rodríguez A, Ortega-Anselmi J, Candel-González FJ, Canora-Lebrato J, Fragiel-Saavedra M, Hernández-Píriz A, et al. Métodos alternativos de CPAP para el tratamiento de insuficiencia respiratoria grave secundaria a neumonía por COVID-19. Med Clin (Barc). 2021;156(2):55-60.

11. Jolliet P, Abajo B, Pasquina P, Chevrolet JC. Non-invasive pressure support ventilation in severe community-acquired pneumonia. Intensive Care Med. 2001;27(5):812-21.

12. Ferrer M, Esquinas A, Leon M, Gonzalez G, Alarcon A, Torres A. Noninvasive ventilation in severe hypoxemic respiratory failure: a randomized clinical trial. Am J Respir Crit Care Med. 2003;168(12):143844.

13. Alhazzani W, Møller MH, Arabi YM, Loeb M, Gong MN, Fan E, et al. Surviving Sepsis Campaign: guidelines on the management of critically ill adults with Coronavirus Disease 2019 (COVID-19). Intensive Care Med. 2020;46(5):854-87.

14. Forrest IS, Jaladanki SK, Paranjpe I, Glicksberg BS, Nadkarni GN, Do R. Non-invasive ventilation versus mechanical ventilation in hypoxemic patients with COVID-19. Infection. 2021;49(5):989-97.

15. Ashish A, Unsworth A, Martindale J, Sundar R, Kavuri K, Sedda L, et al. CPAP management of COVID-19 respiratory failure: a first quantitative analysis from an inpatient service evaluation. BMJ Open Respir Res. 2020;7(1):e000692.

16. Grieco DL, Menga LS, Cesarano M. Effect of helmet noninvasive ventilation vs high-flow nasal oxygen on days free of respiratory support in patients with COVID-19 and moderate to severe hypoxemic respiratory failure: The HENIVOT randomized clinical trial: The HENIVOT randomized clinical trial. JAMA. 2021;325(17):1731-43.

17. Winck JC, Scala R. Non-invasive respiratory support paths in hospitalized patients with COVID-19: proposal of an algorithm. Pulmonology. 2021;27(4):305-12.

18. Faraone A, Beltrame C, Crociani A, Carrai P, Lovicu E, Filetti S, et al. Effectiveness and safety of noninvasive positive pressure ventilation in the treatment of COVID-19-associated acute hypoxemic respiratory failure: a single center, non-ICU setting experience. Intern Emerg Med. 2021;16(5):1183-90.

19. Di Domenico SL, Coen D, Bergamaschi M. Clinical characteristics and respiratory support of 310 COVID-19 patients, diagnosed at the emergency room: a single-center retrospective study. Intern Emerg Med. 2021;16(4):1051-60.

20. Bellani G, Grasselli G, Cecconi M, Antolini L, Borelli M, De Giacomi F, et al. Noninvasive ventilatory support of patients with COVID-19 outside the intensive care units (WARd-COVID). Ann Am Thorac Soc. 2021;18(6):1020-6. 
21. Suardi LR, Pallotto C, Esperti S, Tazzioli E, Baragli F, Salomoni E, et al. Risk factors for noninvasive/invasive ventilatory support in patients with COVID-19 pneumonia: A retrospective study within a multidisciplinary approach. Int J Infect Dis. 2020;100:258-63.

22. De Vita N, Scotti L, Cammarota G, Racca F, Pissaia C, Maestrone C, et al. Predictors of intubation in COVID-19 patients treated with out-of-ICU continuous positive airway pressure. Pulmonology. Published online 2021.

23. Avdeev SN, Yaroshetskiy Al, Tsareva NA, Merzhoeva ZM, Trushenko NV, Nekludova GV, et al. Noninvasive ventilation for acute hypoxemic respiratory failure in patients with COVID-19. Am J Emerg Med. 2021;39:154-7.

24. Liu L, Xie J, Wu W, Chen H, Li S, He H, et al. A simple nomogram for predicting failure of noninvasive respiratory strategies in adults with COVID-19: a retrospective multicentre study. Lancet Digit Health. 2021;3(3):e166-74.

25. Rao KS, Singhai P, Salins N, Rao SR. The pathway to comfort: Role of palliative care for serious COVID-19 illness. Indian J Med Sci. 2020;72(95):95-100.

26. Janssen DJA, Ekström M, Currow DC, Johnson MJ, Maddocks M, Simonds AK, et al. COVID-19: guidance on palliative care from a European Respiratory Society international task force. Eur Respir J. 2020;56(3):2002583.

27. JASP Team. JASP (Version 0.16)[Computer Software]; 2021.

28. Pedregosa F, Varoquaux G, Gramfort A, et al. Scikit-learn: Machine Learning in Python. J Mach Learn Res. 2011;12(85):2825-30.

29. Radovanovic D, Coppola S, Franceschi E, Gervasoni F, Duscio E, Chiumello DA, et al. Mortality and clinical outcomes in patients with COVID-19 pneumonia treated with non-invasive respiratory support: A rapid review. J Crit Care. 2021;65:1-8.

30. Chung H, Ko H, Kang WS, Kim KW, Lee H, Park C, et al. Prediction and feature importance analysis for severity of COVID-19 in South Korea using artificial intelligence: Model development and validation. J Med Internet Res. 2021;23(4):e27060.

31. Kurtz P, Bastos LSL, Dantas LF, et al. Evolving changes in mortality of 13,301 critically ill adult patients with COVID-19 over 8 months. Intensive Care Med. 2021;47(5):538-48.

32. Williams S, Sheard N, Stuart B, Phan HTT, Borca F, Wilkinson TMA, et al. Comparisons of early and late presentation to hospital in COVID-19 patients. Respirology. 2021;26(2):204-5.

\section{Figures}




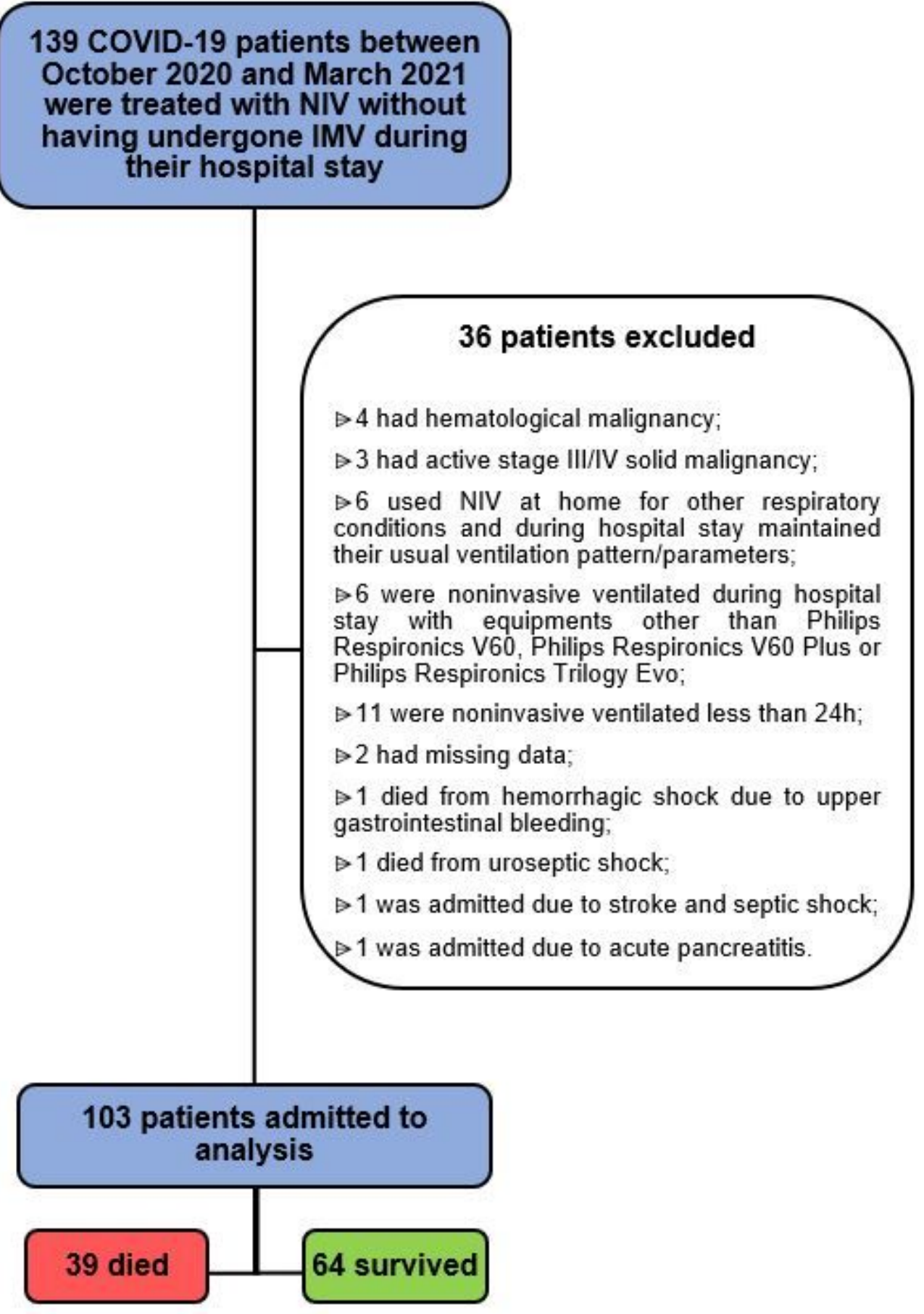

Figure 1

Flowchart of Inclusion and Exclusion of Participants. 139 COVID-19 patients were identified between the period of $1^{\text {st }}$ October 2020 and $31^{\text {st }}$ of March 2021 which were treated with NIV without being candidates for IMV or needing IMV. Applying exclusion criteria 103 patients were admitted to analysis, of which 39 died during the hospital stay. 


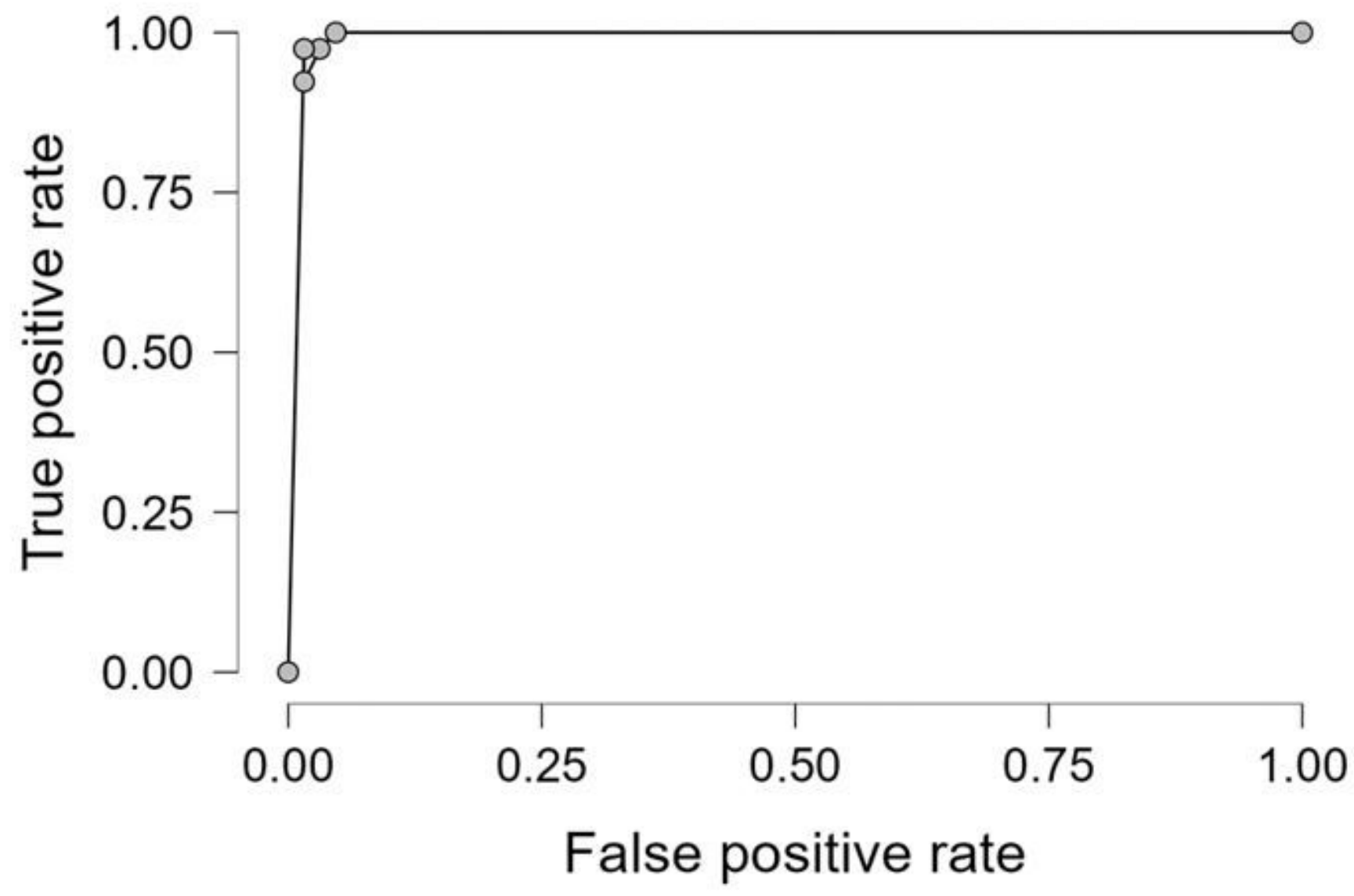

Figure 2

Receiver Operating Characteristic (ROC) Curve for the Predictive Model. The ROC Curve for the Predictive Model has an AUC of 0.994. 


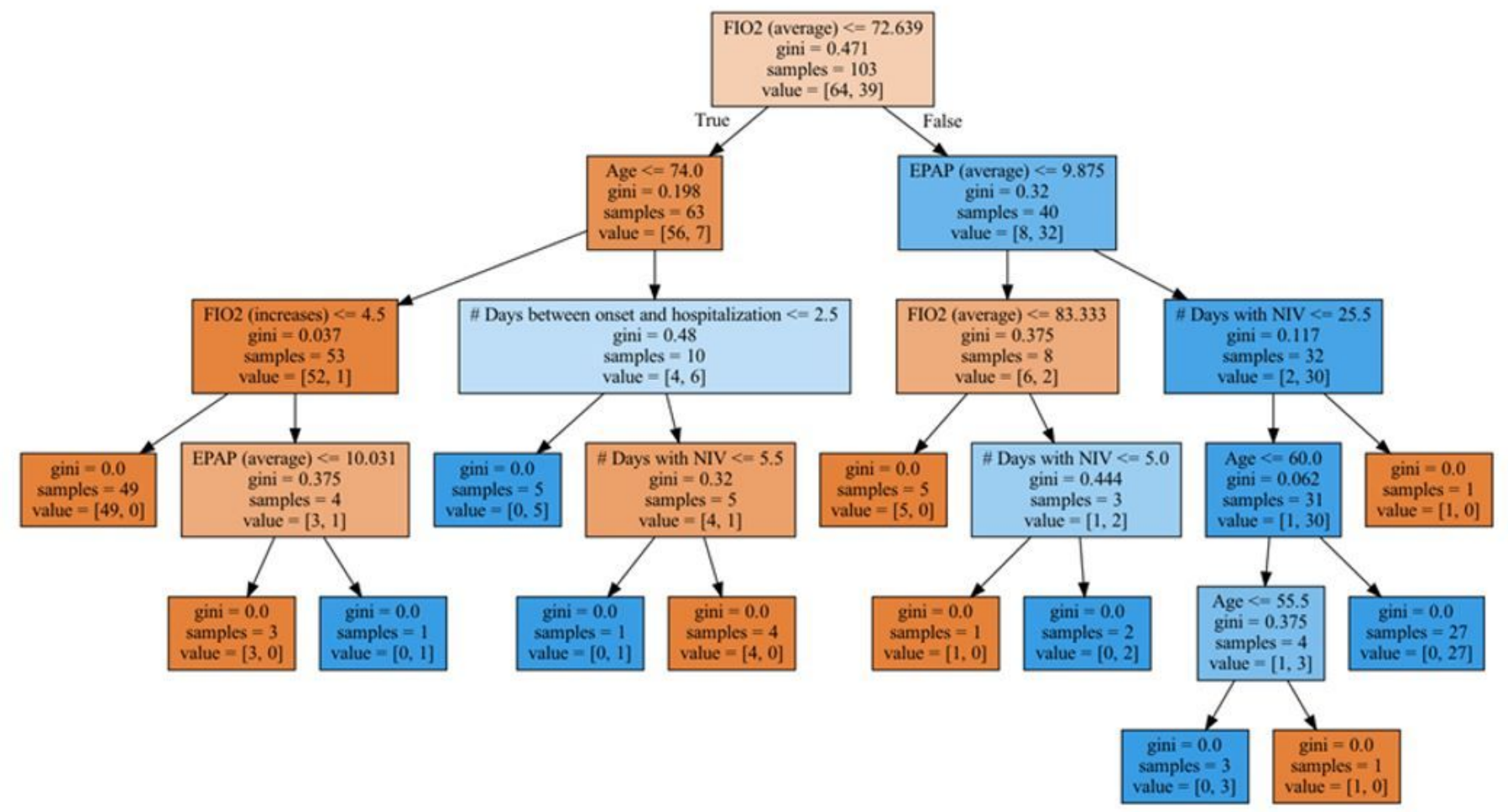

\section{Figure 3}

The Decision-tree Classification Model presented starts with a root node in which all the observations (ntotal $=103$; nalive $=64$; ndead $=39$ ) are present. The first decision boundary was based on the average FiO2 values: approximately $82 \%$ of the patients who die have average $\mathrm{FiO} 2$ values greater than $72.6 \%$. A new decision boundary is defined by age in the group of patients with average FiO2 values equal or lower than $72.6 \%$. From a total of 7 patients in this branch, 6 patients with age greater than 74 years old die. Among the group of patients with average $\mathrm{FiO} 2$ values greater than $72.6 \%$, from a total of 32 patients, 30 patients with EPAP average values greater than 9.9 die. Third level decision boundaries are established according to FiO2 average values, number of days with increases on $\mathrm{FiO} 2$, number of days between disease onset and hospitalization and the total number of days on NIV. 